\title{
Simulation of the Acoustic Field of Broadband Parametric Sonar
}

\author{
S.W. Li \\ Shanghai Marine Electronic Equipment Research Institute \\ Shanghai, 201108, China
}

\begin{abstract}
One of the advantages of parametric array is its broadband ability at low frequency. Recently, parametric acoustic source is gradually being used in communication sonar and detection sonar in shallow water, and broadband signal design for parametric array is pressing in order to obtain high transmitting rate for digital signal communication and good performance for detection in shallow water where reverberation is usually severe. In this paper, Full wave simulation of the acoustic field of broadband parametric sonar is presented, and FDTD (finite-difference time-domain) method is used in producing the simulation. The suggested envelopes according to self-demodulation theory and physical parameters of a parametric sonar are introduced into the full wave simulation. An improved method of generating LFM (Linear Frequency Modulation) signal and a simple method of generating Ricker wavelet are proposed. And for digital signal transmitting, simulation results show that PAM (Pulse Amplitude Modulation) signal are better than BPSK (Binary Phase shift Keying) signal when used as the envelope of primary wave.
\end{abstract}

Keywords-broadband parametric sonar; FDTD; selfdemodulation theory

\section{INTRODUCTION}

Parametric array had been one of the most popular research area in underwater acoustics for decades since Westervelt published his famous paper in 1963[1], and parametric array signal processing is still an active research area so far [2][3][4]. Parametric acoustic source is the most important source for high-resolution sub-bottom profiler, and is being used in detection sonar and communication sonar in shallow water gradually [5] [6] [7] [8] [9] [10].

In 1965, Berktay [11] pointed out that the pressure in secondary acoustic field of parametric array is proportional to the second order derivative of the square of the envelope of the primary wave. This theory is called "selfdemodulation" theory and has been widely used in parametric sonar design. According to this theory, Ricker wavelet, which is exactly the second order derivative of Gaussian wave, has been one of the most important signals for parametric sub-bottom profiler [12] [13] [14] [15].

In this paper, previous work [16] using four different envelopes of primary wave to generate LFM signal, digital signal and Ricker wavelet according to Berktay's theory is reviewed in section II. Because Berktay's self-demodulation theory is an approximate theory [5], in section III, a full wave simulation of the acoustic field of parametric array is produced by using FDTD (finite-difference time-domain) method, and Khokhlov-Zabolotskaya-Kuznetsov nonlinear parabolic equation is used as the model equation for the simulation. Section IV is the summary.

\section{IIANALYSES BASED ON BERKTAY'S THEORY [16]}

Pulsed wave traveling along $z$-direction at time $t$ and distance $\mathrm{z}$ can be represented in the form

$$
p(t, z)=P e^{-\alpha_{1} z} f\left(t-\frac{z}{c}\right) \cos \left[\omega_{0}\left(t-\frac{z}{c}\right)\right]
$$

Where $\mathrm{P}$ is the pressure amplitude and $f(t-z / c)$ is the envelope function whose highest frequency component is very small compared to the carrier frequency $\omega_{0}, \alpha_{0}$ is the absorption coefficient at frequency $\omega_{0}$. From Berktay's theory, the on-axis pressure of difference frequency wave at distance $R$ in the far field can be expressed as:

$$
p_{-}(R, t)=A \frac{\partial^{2}}{\partial t^{2}}\left[f^{2}\left(t-\frac{R}{c}\right)\right]
$$

Where $\mathrm{A}$ is a constant related to the amplitude and frequency of the acoustic wave, absorption of the medium, and aperture of the transducer.

\section{A. LFM Signal}

LFM signal with central frequency $\omega_{\mathrm{d}}$, bandwidth $\Delta \omega$ and pulse length $\Delta \mathrm{T}$ can be expressed as:

$$
f(t)=\sin \left(\omega_{d} t+0.5 \mu t^{2}\right)
$$

Where $\mu=\Delta \omega / \Delta \mathrm{T},-\Delta \mathrm{T} / 2 \leq \mathrm{t} \leq \Delta \mathrm{T} / 2$.

Substituting equation (3) into equation (2) we have:

$$
\begin{aligned}
& p_{-}(R, t)=A \cdot 2\left(\omega_{d}+\mu t\right)^{2} \cos \left[2\left(\omega_{d} t+0.5 \mu t^{2}\right)\right] \\
& +A \bullet \mu \sin \left[2\left(\omega_{d} t+0.5 \mu t^{2}\right)\right]
\end{aligned}
$$

Improved method will use the signal expressed as following equation as envelope of primary wave:

$$
f_{1}(t)=\sin \left(\omega_{d} t+0.5 \mu t^{2}\right) /\left(\omega_{d}+\mu t\right)=f(t) /(g(t)
$$

Here $\mathrm{g}(\mathrm{t})=\omega \mathrm{d}+\mu \mathrm{t}$.

Substituting equation (5) into equation (2), we have

$$
\begin{aligned}
p_{-}(R, t) & =A \bullet 2 \cos \left(2 \omega_{d} t+\mu t^{2}\right) \\
& -A \bullet \frac{3 \mu}{\left(\omega_{d}+\mu t\right)^{2}} \sin \left(2 \omega_{d} t+\mu t^{2}\right) \\
& -A \bullet \frac{6 \mu^{2}}{\left(\omega_{d}+\mu t\right)^{4}} \cos \left(2 \omega_{d} t+\mu t^{2}\right)
\end{aligned}
$$

Neglecting the insignificant terms, we have: 


$$
p_{-}(R, t) \approx A \bullet 2 A \cos \left[2\left(\omega_{d} t+0.5 \mu t^{2}\right)\right]
$$

\section{B. BPSK Signal}

BPSK signal can be expressed as:

$$
f(t)=\cos \left(\omega_{d} t+i \frac{\pi}{2}\right), \quad i=-1, \quad 1, \quad 0<t<T_{s}
$$

Here $T_{s}$ is the period of cosine signal with frequency $\omega_{d}$. The self-demodulation results using BPSK wave as envelope of primary wave will cause spikes in the selfdemodulated signal at the points where the abrupt phase changes occur on the BPSK envelope [15]. Smith [17] suggested a double sideband modulation method to transmit BPSK signal by parametric array, but the bandwidth efficiency was relatively low for that method.

\section{PAM Signal}

From equation (8) and the following equation (9), we can see that BPSK signal is equal to PAM signal with symbols $\mathrm{a}_{\mathrm{i}}= \pm 1$. PAM signal can be expressed as:

$$
f(t)=a_{i} \cos \left(\omega_{d} t\right)
$$

$a_{i}$ is integer, $0<t<T_{s}, T_{s}$ is the period of cosine signal with frequency $\omega_{d}$.

\section{Ricker Wavelet}

Using half-cosine wave through a low-pass filter (to remove the high frequency component caused by the truncation of a cosine wave) as envelope is almost as good as using Gaussian wave, and choosing parameter of half cosine wave is much simpler.

\section{FULL WAVE SIMULATION OF ACOUSTIC FIELD USING FDTD METHOD}

\section{A. Model}

The numerical experiment is based on solving nonlinear acoustic wave equation using FDTD method. One of the accurate mathematical models for a directive sound beam in a thermoviscous fluid is KZK equation. After normalization, the equation can be written as [18]:

$$
\frac{\partial P}{\partial \sigma}=\frac{1}{4(1+\sigma)} \int_{-\infty}^{\tau}\left(\frac{\partial^{2} P}{\partial \rho^{2}}+\frac{1}{\rho} \frac{\partial P}{\partial \rho}\right) d \tau^{\prime}+A \frac{\partial^{2} P}{\partial \tau^{2}}+\frac{N P}{(1+\sigma)} \frac{\partial P}{\partial \tau}
$$

Here $\mathrm{P}, \sigma, \rho$ and $\tau$ are normalized variables, $\mathrm{A}$ and $\mathrm{N}$ are constants. The definition of these variables and constants are:

$\sigma=\mathrm{z} / \mathrm{z}_{0}$, where $\mathrm{z}$ is the coordinate along the axis of the sound beam, $z_{0}=\omega_{0} \mathrm{a}^{2} / 2 \mathrm{c}_{0}$ is the Rayleigh distance for $\mathrm{a}$ circular planar transducer with radius a at angular frequency $\omega_{0}$, and $\mathrm{c}_{0}$ is the ambient sound velocity.

$\mathrm{P}=(1+\sigma)\left(\mathrm{p} / \mathrm{p}_{0}\right)$, where $\mathrm{p}$ is the pressure on the surface of transducer and $\mathrm{p}_{0}$ is the amplitude;

$\rho=(\mathrm{r} / \mathrm{a}) /(1+\sigma)$, where $\mathrm{r}$ is the transverse radial coordinate; time.
$A=\alpha_{0} Z_{0}, N=Z_{0} / z$, where $\alpha_{0}$ is the absorption coefficient of the medium and $z$ ' is the plane-wave shock formation distance when the pressure amplitude is $\mathrm{p}_{0}$ and frequency is $\omega_{0}$. The relative importance of absorption and nonlinearity is determined by the parameter $\Gamma=\mathrm{N} / \mathrm{A}$.

The three terms at the right side of Equation (8) account for diffraction, absorption and nonlinearity, respectively. For each small range step, these three effects are assumed operating independently and are considered separately when solving the equation numerically. If the range step is small enough, the numerical error caused by this assumption is tolerable. The so-called Texas code [18][19][20] for solving axis-symmetric sound field is used for our simulation.

\section{B. Parameters}

Based on the specification of actual parametric sonar, the physical parameters chosen for the numerical experiment are:

$$
\begin{aligned}
& \mathrm{a}=0.25 \mathrm{~m} ; \\
& \mathrm{f}_{0}=\omega_{0} / 2 \pi=80 \mathrm{kHz} \\
& \mathrm{p}_{0}=85197 \mathrm{~Pa}(\text { Source level is } 336 \mathrm{~dB}, \text { ref. } 1 \mu \mathrm{Pa},) \\
& \mathrm{z}_{0}=10.47 \mathrm{~m} ; \\
& \mathrm{z}^{\prime}=141.5 \mathrm{~m} \\
& \alpha_{0}=25 \mathrm{~dB} / \mathrm{km}=0.00288 \text { neper (sea water of salinity } 35 \%
\end{aligned}
$$
at temperature of $15^{\circ} \mathrm{C}$ )

$$
\mathrm{A}=\alpha_{0} \times \mathrm{z}_{0}=0.03 ; \mathrm{N}=\mathrm{z}_{0} / \mathrm{z}^{\prime}=0.07
$$

The source condition is written in the form

$$
\mathrm{p}=\mathrm{p}_{0} \mathrm{~F}(\mathrm{r}, \mathrm{t}) \quad \text { at } \mathrm{z}=0
$$

For uniform actuation force on transducer, $F(r, t)=F(t)$ when $r \leq a, F(r, t)=0$ when $r>a$.

In our numerical experiment, $F(t)=f(t) \cos \left(\omega_{0} t\right)$ at $z=0$, where $f(t)$ is the envelope function. The bandwidth is $2.5 \sim 7.5 \mathrm{kHz}$ and pulse width is $0.5 \mathrm{~ms}$ when LFM signal is used as the envelope, the carrier frequency is $8 \mathrm{kHz}$ and code sequence is $[1,0,0,1,0,1]$ when PAM and BPSK signals are used as the envelopes, and the pulse width (zero to zero) is $1 / 4000 / 2=0.125 \mathrm{~ms}$ (half period of $4 \mathrm{kHz}$ cosine wave) when half cosine signal is used as envelope.

The non-physical parameters for the FDTD simulation method are as follows: 120 points/cycle for time sampling; 60 points across piston for space sampling; step size is $1 . \mathrm{e}^{-3}$ for the first 100 steps along $\mathrm{z}$ coordinate when IBFD (implicit backward finite difference) algorithm is used, and is $3.5 \mathrm{e}^{-3}$ after the first 100 steps when CNFD (CranckNicolson finite difference) algorithm is used.

\section{Results and Discussion}

Amplitudes of all the signals in Fig.1 Fig. 5 are normalized by the source level.

Fig. 1 shows the on-axis difference frequency signals (raw signals through a low-pass filter to remove the primary signal) at distance of $1047 \mathrm{~m}\left(100 \mathrm{z}_{0}\right)$. Fig. 1(a) is the result using LFM signal divided by its instantaneous frequency as envelope of primary wave. We can see that the lower 
frequency component are over compensated; LFM signal divided by the square root of its instantaneous frequency is used as the envelope for Fig. 1(b), and the compensation is deficient; LFM signal divided by the 0.75 th power of its instantaneous frequency is used as the envelope for Fig. 1(c), and the demodulated signal is more approaching to a perfect LFM signal.

Fig. 2(a), (b), (c), (d) shows the on-axis raw signal at the distance of $1047 \mathrm{~m}, 5235 \mathrm{~m}, 10470 \mathrm{~m}$ and $20940 \mathrm{~m}$, respectively. We can see that except for the distance of $1047 \mathrm{~m}$, the low-pass filter is not necessary to extract difference frequency component from the raw signal because the primary signal has been attenuated severely and are almost unobservable at those three further distances.

Fig. 3 Fig. 5 show the difference frequency waves at the four distances mentioned above when the envelope of primary wave is PAM signal, BPSK signal, and half cosine signal, respectively.

Although there are distortions for the PAM signals in Fig. 3 , it is apparent that the code sequence $[1,0,0,1,0,1]$ is quite easy to be extracted from these signals. But for Fig. 4, the difference frequency signals do not look like BPSK signal at all, and BPSK signal demodulation algorithm cannot extract the right code sequence from these signals. From the signals in Fig. 5 it can be observed that the right shoulders of the waveforms are a bit lower than the left ones, unlike a perfect Ricker wavelet whose two shoulders are of the same height. This may change the zero-phase characteristic of Ricker wavelet. The same result was also obtained by using Gaussian wave as envelope and was found in an early experiment ${ }^{[12]}$. Proper explanation for this result is not obtained yet.
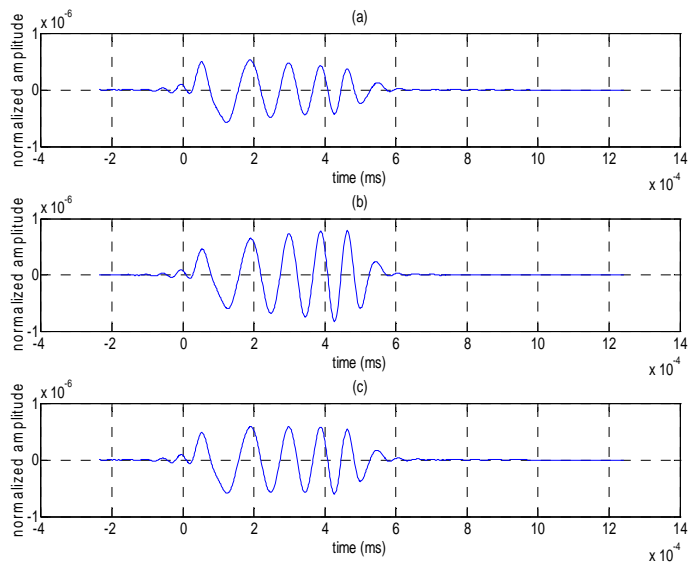

FIGURE I. ON-AXIS DIFFERENCE FREQUENCY SIGNALS AT DISTANCE OF 1047M WHEN ENVELOPES OF PRIMARY WAVE ARE:

(A) LFM SIGNAL DIVIDED BY THE INSTANT FREQUENCY; (B) LFM SIGNAL DIVIDED BY THE SQUARE ROOT OF THE INSTANT FREQUENCY; (C) LFM SIGNAL DIVIDED BY THE 0.75TH POWER OF THE INSTANT FREQUENCY.

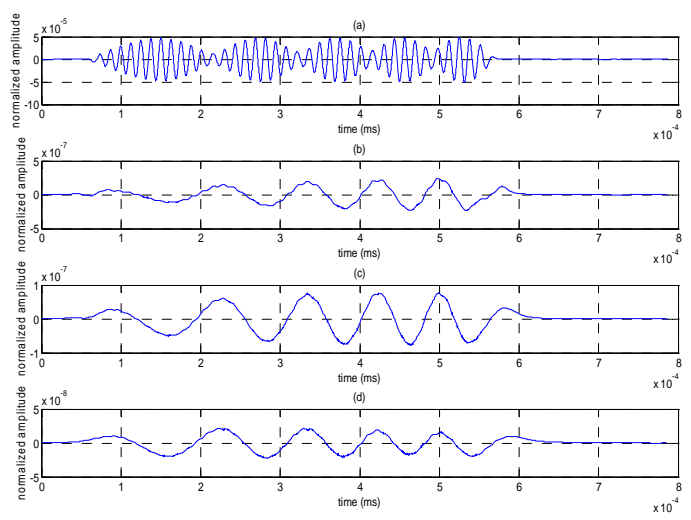

FIGURE II.ON-AXIS RAW SIGNAL AT DISTANCE OF: (A) 1047 M; (B) $5235 \mathrm{M}$; (C) $10470 \mathrm{M}$; (D) 20940 M ENVELOPE OF PRIMARY WAVE IS LFM SIGNAL.

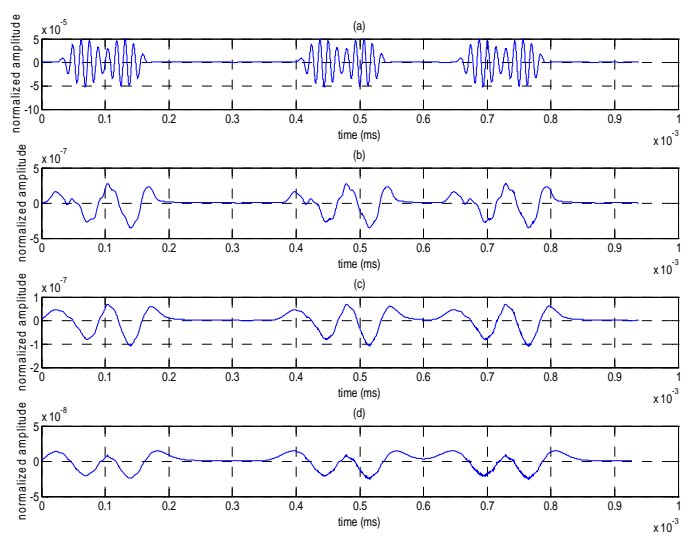

FIGURE III.ON-AXIS RAW SIGNAL AT DISTANCE OF: (A) $1047 \mathrm{M}$; (B) $5235 \mathrm{M}$; (C) $10470 \mathrm{M}$; (D) 20940 M ENVELOPE OF PRIMARY WAVE IS PAM SIGNAL.

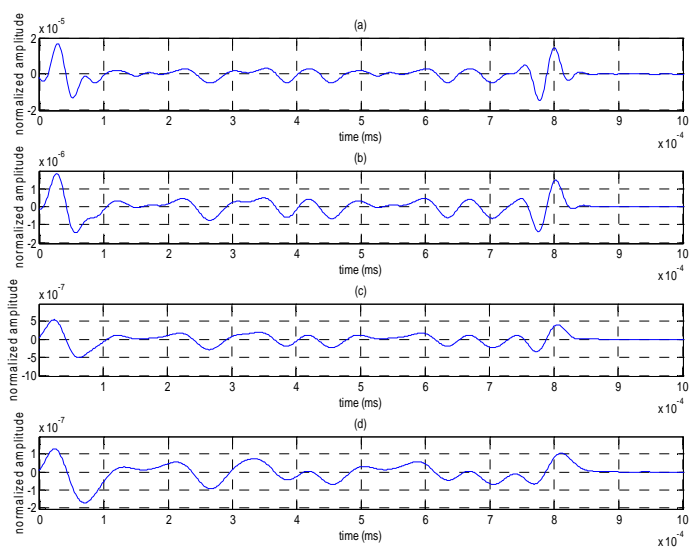

FIGURE IV.ON-AXIS DIFFERENCE FREQUENCY SIGNAL AT DISTANCE OF: (A) 1047 M; (B) 5235 M; (C) 10470 M; (D) 20940 M ENVELOPE OF PRIMARY WAVE IS BPSK SIGNAL. 


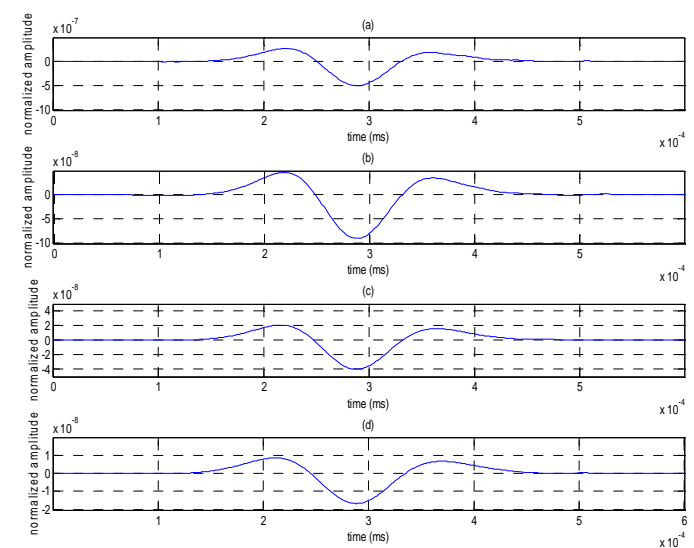

FIGURE V.ON-AXIS DIFFERENCE FREQUENCY SIGNAL AT DISTANCE OF: (A) 1047 M; (B) 5235 M; (C) 10470 M; (D) 20940 M. ENVELOPE OF PRIMARY WAVE IS HALF COSINE SIGNAL.

\section{SUMMARY}

Numerical experiment based on full wave simulation produced by solving accurate nonlinear acoustic equation numerically using FDTD method supports the results of Berktay's theory in general. Full wave simulation results show that not only the nonlinear self-demodulation process, but also the absorption effect should be considered when choosing the envelope of primary wave to generate broadband difference frequency signal for parametric sonar design.

\section{REFERENCES}

[1] P.J. Westervelt, Parametric acoustic array, J. Acoust. Soc. Am. 35 (1963), 535-537.

[2] K. G. Foote,D. T. I. Francis, P. R. Atkins, Calibration sphere for lowfrequency parametric sonars, J. Acoust. Soc. Am. 121(2007), 13241331.

[3] H. Woodsum, W. Hogan, Fourier-operator methods for parametric array analysis: Theory versus experiment, J. Acoust. Soc. Am. 121(2007), 3060.

[4] R. Waxler, T. G. Muir, A theory of low frequency parametric arrays in shallow water, J. Acoust. Soc. Am. 121(2007), 3060.

[5] R. F. W. Coates et al, "BASS 300 PARACOM": A "MODEL" Underwater Parametric Communication System, IEEE J. Oceanic Eng. 21(1996), 225-232.

[6] M.Zheng, L.Wang, R. Stoner, R.F.W.Coates, Underwater digital communication utilizing parametric sonar with M-ary DPSK modulation, Sonar Navig, 146(1999), 213-218.

[7] L. Kopp, D. Cano, E. Dubois, L.S. Wang, B. Smith, R. F. W. Coates, Potential performance of parametric communications, IEEE J. Oceanic E. 25(2000), 282-295.

[8] R. Galvin, L.S. Wang, Measured channel characteristics and the corresponding performance of an underwater acoustic communication system using parametric transduction, Sonar Navig., 147(2000), 247253.

[9] ATLAS PARASOUND DS-3, www.atlashydro.com.

[10] Waterside Security and Asset, http://www.wifihut.com.

[11] H.O. Berktay, Possible exploitation of nonlinear acoustics in underwater transmitting applications. J. Sound Vib. 2(1965), 435-461.

[12] J. Dybedal, A. Løvik, O. Malmo, The parametric array source and application of signal processing, Ultrasonic Symposium, IEEE, 1987, pp. $735-740$.
[13] O. Bergem, N.G. Pace, Installation and calibration of a parametric array for shallow water backscatter measurements, Oceans' 96, MTS/IEEE, 1996, pp. 773-777.

[14] A. Caiti, O. Bergem, J. Dybedal, Parametric sonars for seafloor characterization, Meas. Sci. Technol. 10 (1999) 1105-1115.

[15] J. A. Fawcett: Scattering from a partially fluid-filled, elastic-shelled sphere, J. Acoust. Soc. Am. 109 (2), 508-513, 2001

[16] S. Li, Pre-processing methods for parametric array to generate wideband difference frequency signals. Oceans' 08, MTS/IEEE, 2008

[17] Smith, B.V.: Conditions for distortionless underwater communications using parametric array, Acoustica, 81, 161-166, 1995;

[18] Y.-S. Lee, M. F. Hamilton, Time-domain modeling of pulsed finiteamplitude sound beam. J. Acoust. Soc. Am. 97(1995), 906-916.

[19] Y.-S. Lee, Numerical solution of the KZK equation for pulsed finite amplitude sound beams in thermoviscous fluids, Ph.D. dissertation, The University of Texas at Austin, 1993

[20] R. O. Cleveland, M. F. Hamilton, D. T. Blackstock, Time-domain modeling of finite-amplitude sound in relaxing fluids, J. Acoust. Soc. Am. 99(1996), 3312-3318. 